\title{
A Sensitivity Analysis on Tidal Stream Turbine Loads Caused by Operational, Geometric Design and Inflow Parameters
}

\author{
T.M Nevalainen*, C.M Johnstone, A.D Grant \\ Energy Systems Research Unit, Mechanical 63 Aerospace Engineering Dept., \\ University of Strathclyde \\ 75 Montrose Street, Glasgow, G1 1XJ, UK
}

\begin{abstract}
This paper presents a sensitivity analysis on a numerical tidal stream turbine model where a multitude of input parameters' effect on the load output were determined. The statistical procedure used, known as the Morris method, provided insight into the interactions between parameters as well as showing their comparative influence on the turbine loading. The investigation covered parameters from the operational, geometric design and inflow variable domains where the rotor radius, current shear, blade root pitch, surface velocity and wave height were identified as most influential. The blade pitch was regarded as a surprisingly prominent influence on the loads. The turbine's operating depth and the blade geometry were also found to be of limited influence in the ranges investigated. In terms of load transmission into the internal components of a turbine's drive train, the rotor out-of-plane bending moment, or eccentric bending moment, was found to be considerable contribution to the off-axis loads on the shaft. Therefore, special attention was paid to the input parameters' relationship to the eccentric load component by performing a detailed study on the load variations caused by the identified primary input parameters. It is concluded that performing a sensitivity analysis on a tidal stream turbine in a specific operating climate can yield insight to the expected load range and that the eccentric loading transmitted to the shaft is significant for most input cases.
\end{abstract}

Keywords: BEMT, Sensitivity analysis, Tidal Stream Turbine, Structural loading, Hydrodynamics, Shaft loading

\section{Introduction}

As the development of tidal stream turbines (TSTs) draws ever closer to the deployment of full scale arrays, such as the MeyGen project off the north coast of Scotland [1], the cost and durability of the individual turbine units will be an important factor if the industry is to gain commercial acceptance.

It is reasonable to believe that TSTs will be highly susceptible to component fatigue failures due to the cyclic stresses brought on by the variations the in inflow velocity in

\footnotetext{
* Corresponding author

Email address: thomas.nevalainen@strath.ac.uk (T.M Nevalainen)
} 
the marine environment $[2,3,4]$. Furthermore, it is suspected that the non-uniform inflow velocity gradient across a turbine's rotor will cause off-axis, or eccentric, bending moments on the shaft connection which may be translated into the drivetrain causing excessive wear on internal components such as bearings and seals [3].

Many studies have been presented using both experimental $[5,6,7]$ and modelling approaches $[8,4,9]$ to investigate the hydrodynamic loads on TSTs caused by a variety of sources such as waves, currents, yaw-misalignment and turbulence. The measured quantities of most investigations have been the axial shaft thrust, shaft torque and blade root bending moments, typically collected for one single turbine design. Due to the inherent complexity of the physical problem of hydrodynamic loads on TSTs, experimental datasets are usually limited to the variation of only a few parameters. Consequently, there are currently few studies available highlighting what parameters are most influential on turbine load generation globally and there is a lack of standardised comparative methods to determine the relative importance of each of the input parameters [10].

Galloway et al. [11] showed that the maximum load fluctuations for a scale model TST were $175 \%$ of the median for the out-of-plane bending moments, and the corresponding in-plane load variations were $100 \%$ of the median. The study showed that the out-ofplane loading was as much as 9.5 times greater than the in-plane loads and the cyclic loading from the wave action was reported to be a likely source of accelerated fatigue.

The magnitude and variational characteristics of the out-of-plane bending moments applied to the drive shaft of a TST were studied by Tatum et al. [3], who used a CFD and fluid structure interaction approach to present blade and rotor loading time-histories. The results for a turbine operating in a sheared current flow showed a variation in the resultant rotor out-of-plane bending moment of $24 \mathrm{kNm}$ caused by the non-uniform loads over the blades, which would ultimately be transmitted into the drive train. It was also reported that the angular direction of this bending moment had a range as high as $67^{\circ}$, which was speculated to lead to the load being applied over a limited area of the drivetrain seals and bearings, resulting in reduced life expectancy.

Furthermore, a local parameter study on TST loads was presented by Milne et al. [12] in order to quantify the relative importance of the different inflow parameters on the blade loads of a device. The parameters investigated were the mean current speed, shear profile, water depth, hub height, wave height, wave period and turbulence intensity. The study was conducted using a numerical model of a full scale turbine using a commercial Blade Element Momentum Theory (BEMT) package [13]. The study showed that for the parameters investigated, the wave height and period had a dominant influence on the blade loads, whilst the site depth and shear profile also showed influence. The study did however not include the influence of varying the turbine's geometrical parameters or its operating state.

McCann [10] also presented a sensitivity study on the fatigue loading on a TST caused by surface wave-action and inflow turbulence, using a commercial BEMT design code. The study showed that there was a strong correlation between a turbine's blade root fatigue and the turbulence intensity of the flow, although the wave effects could be seen as the dominant factor in the case investigated. The study highlighted the need for a complete exhaustive study on the inflow parameters to determine the relationships of the inflow conditions to the fatigue loading, it is also recommended that other components, such as transmissions and support structures, are investigated.

It is noted that the majority of the experimental and numerical studies presented in 
literature do not take into account the aforementioned non-uniformity of the loads across the rotor plane at any given instance in time. Therefore, these studies tend to neglect the additional out-of-plane bending moments on the rotor, caused by the non-uniform thrust loads, that will be transmitted to the turbine's shaft and internal components.

Based on the identified lack of knowledge on the parameter influence on hydrodynamic loads on TSTs in a global sense, the main aim of this paper is to present a method to rank the importance of the inflow, operational and geometrical parameters of a TST operating in the marine environment and to inform on the relationships between the parameter's and the output loads. Special attention is given to the parameters' influence on the variance of the loads on a turbine's components, as this was considered to be a major driver for the durability and fatigue life of a device. Furthermore, since the studies on TST loading found in literature unanimously show that the rotor thrust is the dominant load on the structure, the focus of this paper will be on the parameters' influence on the thrust load only. Finally, since the non-uniformity of the thrust loads across the rotor plane was found to be an often over-looked aspect of TST loading, the influence of the identified dominant parameter's effect on the eccentric shaft bending moments will be studied in greater detail.

The results of the turbine thrust loads presented in this paper were derived using a numerical BEMT model, adapted for use on TSTs in dynamic inflow conditions. This numerical model was implemented in MATLAB, as originally described in Nevalainen et al. [14], and was based on the general architecture as presented by Masters et al. [9]. The influence the input parameters had on the loads was determined using the Morris method sensitivity analysis [15], where the fast convergence time of the numerical BEMT code was utilised to run the hundreds of model permutations needed for a statistically robust analysis of the load sensitivity. With the most influential input parameters in regards to the turbine loads identified, a detailed analysis of the influence of these 'primary parameters' on the rotor out-of-plane bending moments was performed. This gave insight into the parameters' relationship with the loads, and load variations, on the rotor which is equivalent to the loads on the turbine shaft connection.

Presented below are brief overviews of the numerical modelling techniques used, and the set-up of the sensitivity analysis by the definition of the input parameters and the output metrics studies.

\section{Methods}

This section presents a brief outline of the principles of the unsteady BEMT method used to derive the turbine loads including the modifications made in order to adapt it for TST applications. The general principles of the Morris method sensitivity analysis are also shown here to give the reader a basic understanding of the algorithm. The sensitivity analysis was divided into three different cases, where the first two explored the influence of inflow and geometric design parameters, and the third case explored the parameter influence over the whole input space combining inflow, geometrical and operational parameters. The definitions of the input parameters and the studied output metrics are presented at the end of this section.

The following section gives an outline of the wave-current model used for the inflow synthesis for the BEMT model. 


\subsection{Coupled Wave-Current Inflow Model}

When considering the inflow synthesis of any TST model, it is important to note that studies conducted by Marcus et al. [16] and Wang and Li [17] indicated that the effects of wave-current interactions give as much as $30 \%$ higher maximum loads on submerged structures than the individual wave and current loads superimposed. This suggested that it was appropriate to incorporate a higher fidelity wave-current theory as the inflow model for the BEMT code which was able to resolve the higher order wave-current interaction effects.

For this reason, a third order Stokes wave model coupled with a constant gradient current profile, as developed by Kishida and Sobey [18], was used for the inflow synthesis in this study. This model reproduced the sub-surface velocity orbitals caused by the wave action including the interaction between the waves and the current profile. In addition, the model allowed the slope of the constant gradient current profile to be varied in order to study its effect on the turbine's loads. The wave-current model was based on potential flow theory which limited it to only be applicable to cases where the flow was of a constant vertical vorticity distribution, $\omega_{\mathrm{c}}$, i.e a constant gradient velocity profile with shear steepness $\omega_{\mathrm{c}}$, which was specified by a bottom and surface velocity $U_{b}$ and $U_{s}$ as shown in Figure 2.

Since the wave-current model used in this investigation assumed a steady and linear current profile, the turbulent eddies that would be present in a real tidal flow site are not resolved and their impact on the loads are neglected. However, as was indicated by McCann [10], the contribution of the ambient flow turbulence to the fatigue damage of a TST is likely to be secondary compared to that of the wave action and shear inflow effects.

\subsection{Unsteady Blade Element Momentum Theory}

BEMT was the main numerical model used to derive the hydrodynamic TST loads which were studied in the sensitivity analysis. BEMT has been used extensively in the wind energy sector and has recently been adapted for use in tidal energy applications and detailed descriptions of it can be found in various text books $[19,20]$.

The main principle of the method is to divide each blade into several blade sections where the fluid and blade section forces are equated and solved for. The total forces on the blades are then obtained as the sum of each blade element's thrust and torque contribution. The thrust and torque forces are further dependant on the local inflow angle, $\varphi_{i}$, and inflow vector magnitude, $V_{i}$, over the $i$-th blade section. The original BEMT equations expressing these quantities must be modified to include the varying inflow velocities from the inflow model as

$$
\begin{gathered}
\varphi_{i}=\tan ^{-1}\left(\frac{u_{\mathrm{B} i}(1-a)}{\Omega_{\mathrm{r}} r(1+b)-w_{\mathrm{B} i}}\right) \\
V_{i}=\sqrt{\left(u_{\mathrm{B} i}(1-a)\right)^{2}+\left(\Omega_{\mathrm{r}} r(1+b)-w_{\mathrm{B} i}\right)^{2}}
\end{gathered}
$$

where $u_{\mathrm{B} i}$ is the local axial inflow component at the $i$-th blade section on blade $\mathrm{B}, a$ and $b$ are the axial and radial induction factors, $\Omega_{\mathrm{r}}$ is the rotor angular velocity, $r$ is the blade section radial location and $w_{\mathrm{B} i}$ is the vertical contribution of the wave velocity at the blade section at any given time-step. 


\subsubsection{Dynamic Inflow Modelling}

The standard steady-state BEMT formulations referenced in the previous section assume constant equilibrium between the wake and the rotor plane at every instance in time. As a consequence, any change in the turbine's operating mode or inflow conditions - which will change the pressure at the rotor-plane - will assume that the wake instantaneously reaches the new equilibrium pressure of the rotor. However, experimental data shows that any sudden change in turbine operation causes a temporary load enlargement on the rotor until the wake recovers to the new equilibrium state $[21,22]$. This load overshoot has been attributed to the time-lag of the wake recovery caused by the fluid taking time to accelerate from the speed at the rotor plane to the speed in the wake. This causes a temporary pressure gradient across the rotor which increases the load $[23,24]$. This phenomenon is termed 'dynamic inflow' and has been shown to be a significant factor of temporary load increase in wind turbines.

To resolve this phenomenon for the un-steady conditions investigated in this paper, the fundamental momentum equations in the BEMT code were altered to capture the dynamic wake effects as originally done by Pitt and Peters [25] and GL Garrad Hassan [26, 13]. This was accomplished by giving the standard expression for the thrust coefficient for a single blade section, $C_{\mathrm{FA}}=4 a(1-a)$, a dependency on the time-derivative of the axial induction factor, $\dot{a}$, as

$$
C_{\mathrm{FA}}=4 a(1-a)+\frac{16}{3 \pi U_{0}} \frac{\left(R_{2}^{3}-R_{1}^{3}\right)}{\left(R_{2}^{2}-R_{1}^{2}\right)} \dot{a}
$$

where $U_{0}$ was the inflow velocity at the blade section, and $R_{1}$ and $R_{2}$ were its inner and outer radii. This gave the wake reaction a transient time-dependency related to the changes in the inflow conditions and the operating mode of the turbine, allowing the loads to be more accurately predicted as shown in Nevalainen et al. [14].

\subsubsection{Fluid Acceleration Effects}

A final modification was done to the BEMT code in order to capture the inertial effects the wave-motion. All submerged objects in an oscillating flow field experience drag forces due to adverse pressure gradients and skin friction, and inertia forces due to the acceleration of the fluid. This inertia component is not captured by standard BEMT methods due to the assumption of steady inflow and must therefore be calculated separately and added to the lift and drag forces on the blades.

The most established method of calculating wave-induced inertial forces on submerged objects is the Morison equation [27] which Buckland [28] presented a method of employing on a blade element level for TSTs. This allowed the force distribution caused by the added mass to be resolved along the blade and avoided empirical assumptions regarding the rotor as a whole.

The final expressions for the inertia forces for a blade section in the axial and tangential directions, $\mathrm{d} F_{\mathrm{in}, \text { axial }}$ and $\mathrm{d} F_{\mathrm{in}, \tan }$, were defined as

$$
\begin{aligned}
& \mathrm{d} F_{\text {in }, \text { axial }}=\rho\left(1+\frac{\pi((c \sin \theta) / 2)^{2}}{A_{\alpha}}\right) A_{\alpha} \frac{\partial u_{\mathrm{Bi}}}{\partial t} \mathrm{~d} r \\
& \mathrm{~d} F_{\text {in, } \tan }=\rho\left(1+\frac{\pi((c \cos \theta) / 2)^{2}}{A_{\alpha}}\right) A_{\alpha} \frac{\partial w_{\mathrm{Bi}}}{\partial t} \mathrm{~d} r
\end{aligned}
$$


where $A_{\alpha}$ was the cross sectional area of the airfoil at the blade section, $\rho$ was the fluid density, $c$ was the section chord length, $\theta$ was the blade section angle to the inflow and $\mathrm{d} l$ was the blade section length.

\subsubsection{Calculation of Thrust Load Eccentricity Vector}

For the purpose of showing the off-axis component of the thrust loading of a TST operating in unsteady flow, a thrust eccentricity vector, $V_{\text {ecc }}$, was defined and used in the qualitative interpretation of the analysis of the primary parameters in Section 4. This rotor vector described the direction and magnitude in which the rotor was forced due to the out-of-plane bending moment caused by the non-uniform loading across the turbine's blades. The eccentricity vector was constructed by first allowing the blade's out-of-plane bending moment vectors to be represented by three vectors oriented in the blade's directions shown as $V_{1}, V_{2}$ and $V_{3}$ in Figure 1 . The bending moment vectors were then decomposed to the components $\left(y_{\mathrm{v} 1}, z_{\mathrm{v} 1}\right),\left(y_{\mathrm{v} 2}, z_{\mathrm{v} 2}\right)$ and $\left(y_{\mathrm{v} 3}, z_{\mathrm{v} 3}\right)$, in a shaft-centred inertial coordinate system $\left[y_{1}, z_{1}\right]$ with $z_{1}$ aligned vertically. The eccentricity vector $V_{\text {ecc }}$ was finally constructed as the resultant vector of the three blade's out-of-plane bending moment vectors, with its components being $y_{\mathrm{ecc}}=y_{\mathrm{v} 1}+y_{\mathrm{v} 2}+y_{\mathrm{v} 3}$ and $z_{\mathrm{ecc}}=z_{\mathrm{v} 1}+z_{\mathrm{v} 2}+z_{\mathrm{v} 3}$ as shown in Figure 1.

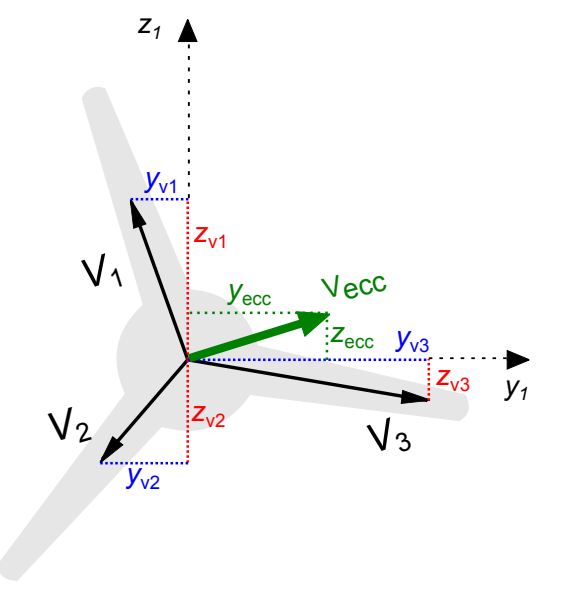

Figure 1: The vectors $V_{1}, V_{2}$ and $V_{3}$, which represented the direction-of-action of the thrust-induced out-of-plane bending moments for each blade, were deconstructed to their components in a shaft-centred coordinate system. Each blade's bending moment-components were then added to create the components $y_{\text {ecc }}$ and $z_{\text {ecc }}$ of the eccentricity vector $V_{\text {ecc }}$.

\subsection{Morris Method Sensitivity Analysis}

As mentioned, the sensitivity analysis performed in this paper used the Morris method, which is given a brief introduction in this section and the full description can be found in Nevalainen et al [29].

Sensitivity analysis is the study of the relationships between the inputs and outputs of a computational model [30], or put more precisely, it is the study of the propagation of variability through the model from the input to the output. Sensitivity analysis can 
provide insight in to model behaviour and performance in extreme cases in addition to determining the model's sensitivity toward its input parameters.

Sensitivity analysis is an established method of load and cost investigations for wind turbines [31,32] and there are several different types of approaches available, each having their own advantages and weaknesses for different applications. A major distinction can be made in sensitivity analysis methods as being either local or global [33], where the former focuses on small perturbations of the input parameters and the latter explores the parameters' full input space.

The Morris method sensitivity analysis [15] used in this study is a global one-at-atime method, meaning that each input parameter was separately varied over its whole assumed input range while the others were kept at a base value. One of the advantages one-at-a-time methods have over methods where all parameters are varied simultaneously is that the information of each parameter's impact on the output is retained and can be studied in detail.

The Morris method is based on the assumption that if all parameters are changed with the same relative amount, the one that causes the largest output change is the most important one. The parameters are then ranked according to their relative importance to each other in a qualitative way.

In a complex computational model such as the unsteady BEMT code, there are likely to be interactions between the different input parameters giving rise to non-linear effects on the output. To achieve statistically significant results and to account for the parameter interactions, the Morris sensitivity analysis works by performing hundreds of permutations on the input-change configurations and storing the results of the inputoutput effects in distributions. The output-changes are then studied in a statistical manner by calculating the absolute mean value, $\mu^{*}$, and standard deviations, $\sigma$ of the distributions. These values are known as the sensitivity indices and are used to rank the output's sensitivity to the individual input parameters. A parameter scoring high on the sensitivity index $\mu^{*}$ shows that it is influential towards the output and a high $\sigma$ score indicates that there is a large inter-parameter relationship present, or that the parameter has a non-linear relation to the output.

\section{Sensitivity Analysis}

Presented in this section is the set-up of the three sensitivity analysis cases and the definition of the investigated parameters and output metrics, followed by the results of the study.

\subsection{Sensitivity Analysis Input Variables and Case Set-Up}

The sensitivity analysis was performed in three separate cases in an attempt to isolate the dominant parameters in the sea-state domain, the geometry design domain and one case concerning all variables to identify the primary parameters in the global domain. The cases are summarised as:

Case 1: Sea-state and operational parameters were varied to investigate the influence on the loads and their interactions, while all other parameters were kept constant. A uniform current profile was assumed in order to isolate the effect of the wave-rotor interactions. The output studied here was the thrust load signal for a single blade. 
Case 2: Turbine geometry and operational parameters were varied while the seastate was kept constant. A uniform current profile was also assumed and the output studied was again the thrust load signal for a single blade.

Case 3: Sea-state, turbine geometry and operational parameters were varied to show the influence of the parameters globally. In this case, a constant gradient velocity profile was assumed to include the effects of the non-uniform inflow across the rotor. The output studied in this case was the eccentricity vector of the rotor.

Figure 2 shows the three domains of sea-state, turbine geometry and operational parameters. The sea-state parameters included in the study were the wave height $H$, wave period $T$, uniform inflow velocity $U$, surface velocity $U_{\mathrm{s}}$, bottom velocity $U_{\mathrm{b}}$, and current constant vorticity $\omega_{\mathrm{c}}$. The operational parameters studied were the rotor angular velocity $\Omega_{\mathrm{r}}$, blade root pitch $\theta$, blade initial azimuth position $\phi$ and hub height $h_{\text {hub }}$. Finally, the turbine geometry parameters studied were the turbine radius $R$, the blade chord distribution defined by the root chord ('Root chord'), and a scalar factor increasing or decreasing the blade's twist distribution ('Twist dist.'). The variable ranges and units for each case are shown in Table 1 and the parameters kept constant over all cases were the water depth, $h$, the water density, $\rho$, and the Reynolds number using the blade chord as characteristic length.

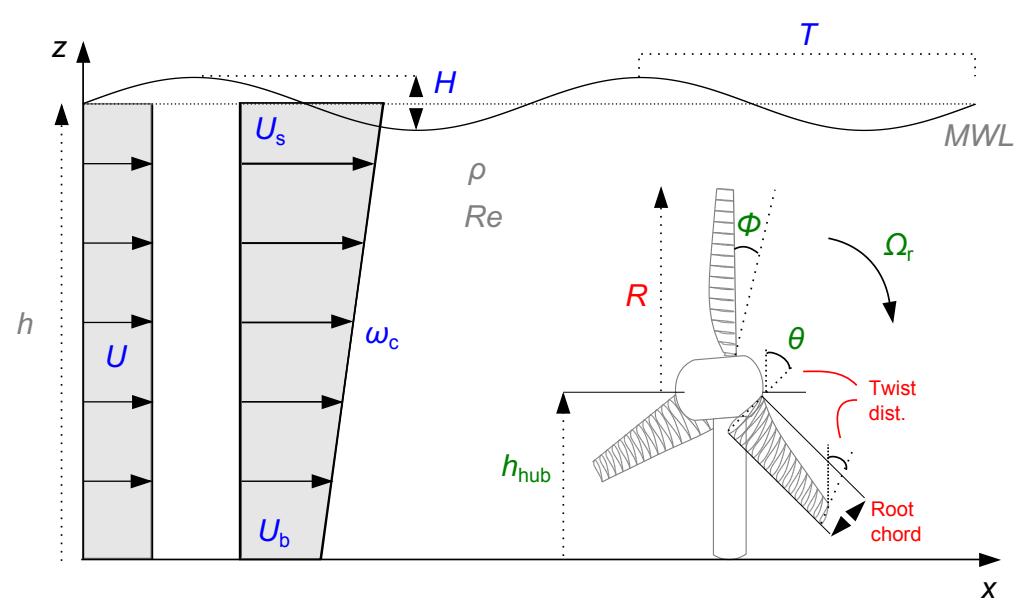

Figure 2: The three domains of the parameters investigated in the sensitivity analysis are the sea-state parameter domain (blue), the turbine operational parameter domain (green) and the turbine geometrical parameter domain (red). The grey parameters are kept constant through all cases. Also shown is the two different inflow current profiles used in the cases, which are used in combination with the Kishida and Sobey inflow model.

The time-step of the simulations were kept at $0.1 \mathrm{~s}$ in order to capture the possible synchronisation effects between the rotor period and wave period. It was believed that certain phase differences between the two parameters could have a significant impact on the loads, which is why the blade initial azimuth angle was included in the study. This time-step size was also large enough to allow the simulations to be completed within a reasonable time-frame, while still being small enough to allow convergence robustness. 
Table 1: Variable ranges investigated in each of the three sensitivity analysis cases. Each group of the table shows which parameters were set to vary and which were kept as constant.

Case 1: Varying sea-state and operational parameters

\begin{tabular}{|c|c|c|c|c|c|c|c|}
\hline Var. & $\begin{array}{c}H[\mathrm{~m}] \\
1-6\end{array}$ & $\begin{array}{c}T[\mathrm{~s}] \\
6-10\end{array}$ & $\begin{array}{c}h_{\mathrm{hub}}[\mathrm{m}] \\
30-40\end{array}$ & $\begin{array}{c}U[\mathrm{~m} / \mathrm{s}] \\
2.5-4\end{array}$ & $\begin{array}{c}\theta\left[^{\circ}\right] \\
-5-5\end{array}$ & $\begin{array}{c}\Omega_{r}[\mathrm{rad} / \mathrm{s}] \\
0.8-1.6\end{array}$ & $\begin{array}{l}\phi[\mathrm{rad} \\
0-2 \pi\end{array}$ \\
\hline Const. & $\begin{array}{c}\rho\left[\mathrm{kg} / \mathrm{m}^{3}\right] \\
1027\end{array}$ & $\begin{array}{c}\text { Blade } \\
\text { sect. } \\
\text { NRELs814 }\end{array}$ & $\begin{array}{c}\text { Root } \\
\text { chord }[\mathrm{m}] \\
3.14\end{array}$ & $\begin{array}{c}\text { Twist } \\
\text { dist. [-] } \\
1\end{array}$ & $\begin{array}{l}\text { Re nr }[-] \\
1.5 \times 10^{4}\end{array}$ & $\begin{array}{c}R[\mathrm{~m}] \\
12.5\end{array}$ & $\begin{array}{r}h[\mathrm{r} \\
70\end{array}$ \\
\hline
\end{tabular}

Case 2: Varying turbine geometry and operational parameters

\begin{tabular}{cccccccc}
\hline Var. & $R[\mathrm{~m}]$ & $\left.\theta 0^{\circ}\right]$ & $\Omega_{r}[\mathrm{rad} / \mathrm{s}]$ & $\begin{array}{c}\text { Root } \\
\text { chord }[\mathrm{m}]\end{array}$ & $\begin{array}{c}\text { Twist } \\
\text { dist. }\end{array}$ & $\phi[\mathrm{rad}]$ & $h_{\text {hub }}[\mathrm{m}]$ \\
& $10-15$ & $-5-5$ & $1.2-1.5$ & $2.5-3.5$ & $0.7-1.3$ & $0-2 \pi$ & $30-40$ \\
& & & & & & \\
Const. & $\rho\left[\mathrm{kg} / \mathrm{m}^{3}\right]$ & $\begin{array}{c}\text { Blade } \\
\text { sect. }\end{array}$ & $H[\mathrm{~m}]$ & $T[\mathrm{~s}]$ & Re nr $[-]$ & $U[\mathrm{~m} / \mathrm{s}]$ & $h[\mathrm{~m}]$ \\
& 1027 & NRELs814 & 3.5 & 8 & $1.5 \times 10^{4}$ & 3.25 & 70
\end{tabular}

Case 3: Varying sea-state, turbine geometry and operational parameters

\begin{tabular}{|c|c|c|c|c|c|c|c|}
\hline Var. & $\begin{array}{c}R[\mathrm{~m}] \\
10-15\end{array}$ & $\begin{array}{c}\theta\left[^{\circ}\right] \\
-5-5\end{array}$ & $\begin{array}{c}\Omega_{r}[\mathrm{rad} / \mathrm{s}] \\
1.2-1.5\end{array}$ & $\begin{array}{c}\text { Root } \\
\text { cord }[\mathrm{m}] \\
2.5-3.5\end{array}$ & $\begin{array}{c}\text { Twist } \\
\text { dist. [-] } \\
0.7-1.3\end{array}$ & $\begin{array}{c}\phi[\mathrm{rad}] \\
0-2 \pi\end{array}$ & $\begin{array}{c}H[\mathrm{~m}] \\
1-6\end{array}$ \\
\hline & $\begin{array}{c}T[\mathrm{~s}] \\
6-10\end{array}$ & $\begin{array}{c}h_{\text {hub }}[\mathrm{m}] \\
30-40\end{array}$ & $\begin{array}{c}U_{s}[\mathrm{~m} / \mathrm{s}] \\
2.5-4\end{array}$ & $\begin{array}{c}\omega_{\mathrm{c}}[\mathrm{m} / \mathrm{s}] \\
0-0.0286\end{array}$ & & & \\
\hline Const. & $\begin{array}{c}\rho\left[\mathrm{kg} / \mathrm{m}^{3}\right] \\
1027\end{array}$ & $\begin{array}{c}\text { Blade } \\
\text { sect. } \\
\text { NRELs814 }\end{array}$ & $\begin{array}{l}\text { Re nr [-] } \\
1.5 \times 10^{4}\end{array}$ & & & & \\
\hline
\end{tabular}

\subsection{Sensitivity Analysis Output Metrics}

The load output studied for Case 1 and Case 2 of the sensitivity analysis was the timehistory of the root thrust for a single blade over the time of two wave periods. Since this model output was a signal composed of a multitude of harmonics, it was necessary to define several statistics to describe the variation and magnitude of the blade loads.

Figure 3a shows the four load metrics studied in Case 1 and Case 2 which were defined as the signal's mean value, its standard deviation, the absolute amplitude of the signal and the fraction of time outside the standard deviation bands. The motivation for the choice of these metrics was that the mean value would represent the total thrust load on the blade while the standard deviation and absolute magnitude would reflect the variation of the signal, which was related to the fatigue loading of the turbine. Finally, the fraction of time outside the standard deviation bands informed on the 'steepness' of the smaller signal harmonics, which also affected the fatigue loading.

Conversely, the output signal studied for Case 3 was the eccentricity vector (Section 2.2.3), which was caused by the non-uniform blade loads and ultimately acted as a offaxis load on the shaft connecting flange. Figure $3 \mathrm{~b}$ shows a polar plot, in-plane with the 
swept rotor area, with the eccentricity vectors plotted for several time-steps. The area covered by the eccentricity vectors was an indicator of the magnitude and directional spread of the out-of-plane bending loads on the rotor. The output metrics defined for this case were the maximum value of the eccentricity magnitude, the arc angle enclosing the load vectors, the standard deviation of the eccentricity vector angles to the vertical, and the number of peaks in the signal shown in Figure 3b.

By using two different sets of output metrics for the three cases, it was possible to obtain information about the parameter's influence on the loads in two degrees of freedom.

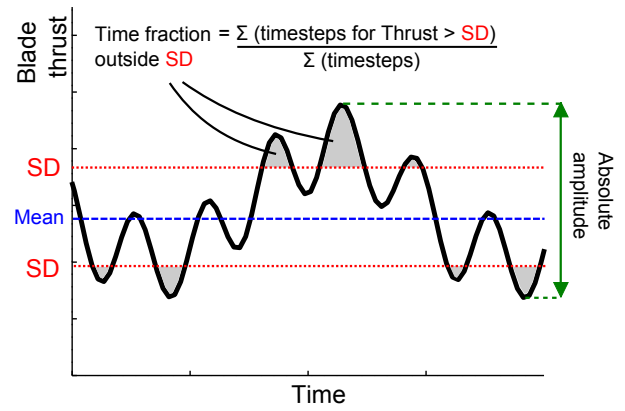

(a)

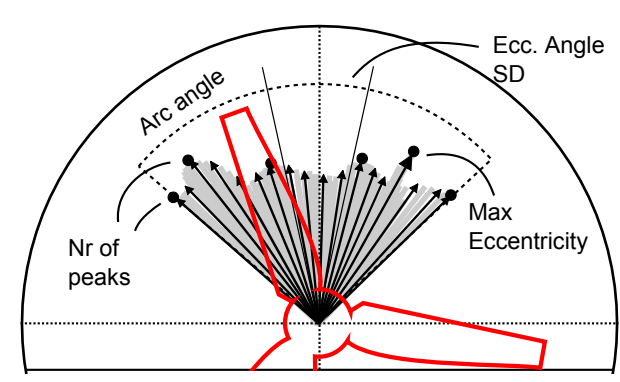

(b)

Figure 3: Definition of the output metrics for the different cases of the sensitivity analysis. For Case 1 and Case 2 (a), the blade root thrust signal was analysed by the four characteristic metrics signal mean, standard deviation (SD), time fraction outside SD and absolute amplitude. For Case 3 (b), the eccentric bending moment pattern was analysed by the characteristic metrics of maximum eccentricity magnitude, enclosing arc angle of the load pattern, number of peaks in the signal and the SD of the vector angles to the vertical.

\subsection{Morris Method Sensitivity Analysis Results and Discussion}

The results of the three sensitivity analysis cases are shown in Figures 4 - 6 respectively. The data is presented so that each of the defined output metrics correspond to one scatter plot in each figure, resulting in four plots per case. The sensitivity of a output metric to the input parameters is shown by the relative sensitivity indices of each parameter, where the $\mu^{*}$ index is plotted against the $\sigma$ index. As an example, Figure 4a shows that the sensitivity of the mean value of the blade thrust signal is most sensitive to the uniform inflow velocity, $U$, indicated by the parameter's high $\mu^{*}$-score. It is recalled that since the sensitivity indices $\mu^{*}$ and $\sigma$ denote the parameter's influence and inter-parameter interactions respectively, the rotor angular velocity, $\Omega_{\mathrm{r}}$, therefore shows highest interaction with other parameters in Figure 4a.

In general, the results in Figures 4 - 6 showed that the loads on a TST were most sensitive to the inflow velocity, wave height, wave period, rotor angular velocity, rotor radius and blade pitch angle. These results were to be expected since these parameters governed the inflow velocities, angles of attack over the blade and power capture area of the turbine, which are all closely linked to the loading. However, further details of the input-output relations can be seen when examining the results of each sensitivity analysis case individually. 


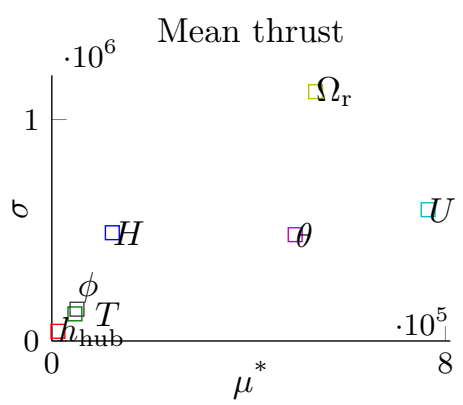

(a)

Time fraction of thrust $>$ SD

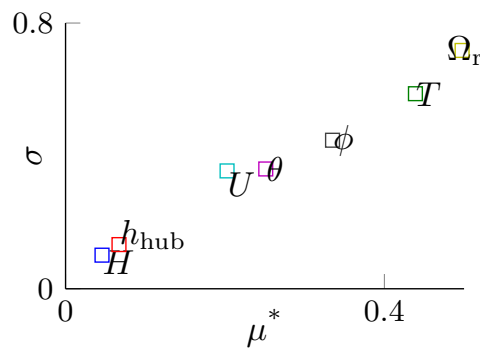

(c)

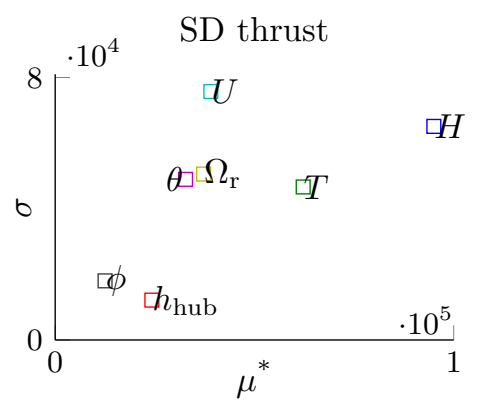

(b)

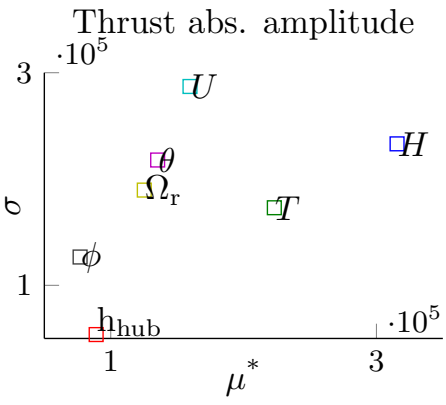

(d)

Figure 4: The results from Case 1 of the sensitivity analysis on the BEMT code showing four scatter plots for the output metrics mean thrust (a), standard deviation (SD) of thrust (b), fraction of time of thrust outside the standard deviation bands (c) and the amplitude of the thrust signal (d). A parameter further to the left on the $\mu^{*}$-axis indicates a higher influence on the loads and a parameter further up on the $\sigma$-axis indicates that it interacts with other parameters.

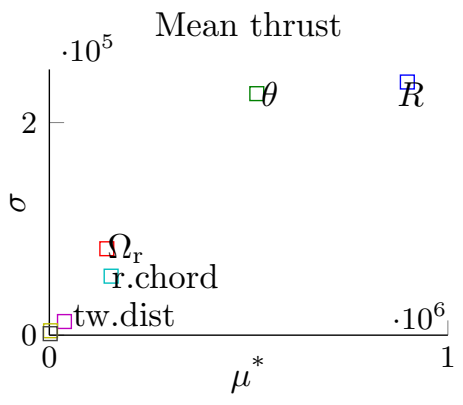

(a)

Time fraction of thrust $>$ SD

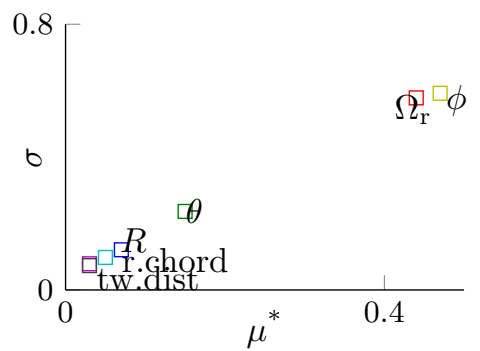

(c)

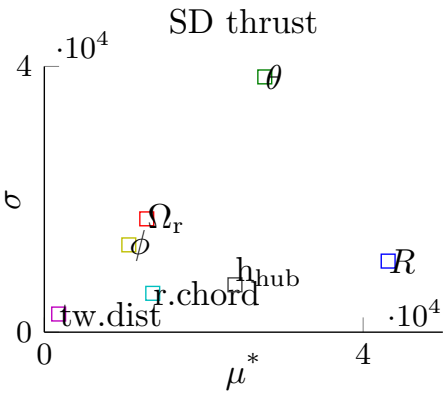

(b)

Thrust abs. amplitude

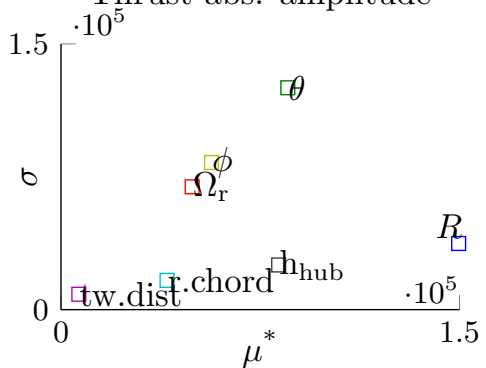

(d)

Figure 5: The results from Case 2 of the sensitivity analysis on the BEMT code showing four scatter plots for the same metrics as in Figure 4. 


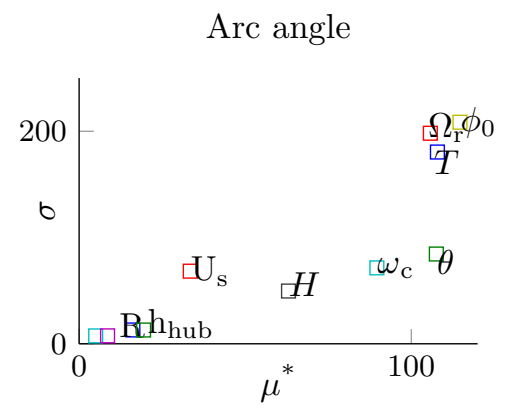

(a)

Eccentric angle SD

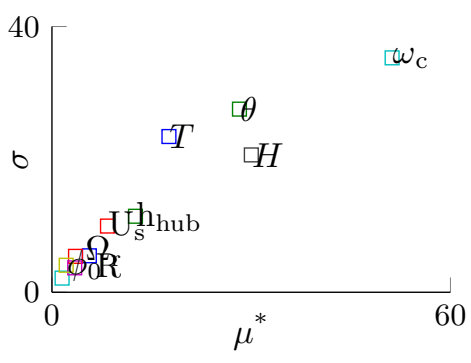

(c)

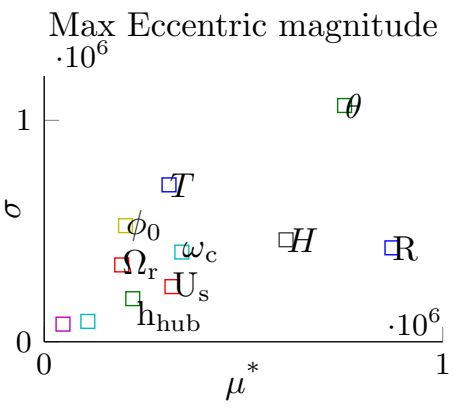

(b)

Number of peaks

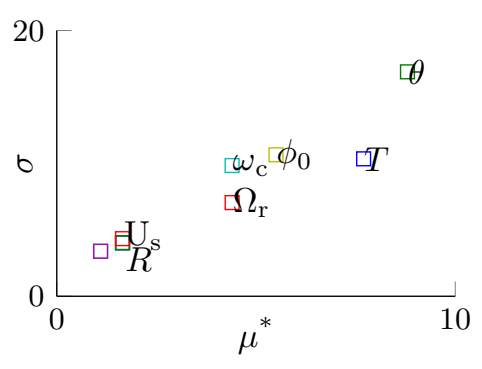

(d)

Figure 6: The results from Case 3 of the sensitivity analysis on the BEMT code showing four scatter plots for the output metrics defined for the eccentricity vector, derived from the out-of-plane bending loads on the rotor. The metrics studied were the load pattern's arc angle (a), max eccentric magnitude (b), eccentric angle standard deviation (SD) (c) and the number of peaks in the load signal (d). Figure $3 \mathrm{~b}$ shows the description of the above metrics. 
Figure 4a shows that the current velocity, rotor angular velocity and blade pitch were the dominant parameters in regards to the mean thrust load in Case 1 exploring the sea-state domain. This is believed to be caused by the parameters altering the local inflow angle over the blade in Equation (3), which in turn has a strong influence on the blade thrust.

Since the rotor angular velocity and blade pitch of a TST can be changed depending on what power control-scheme is used, the mean blade loads could be managed by optimising the trade-off between the turbine's power capture and blade-loading. On the other hand, Figures $4 \mathrm{~b}-4 \mathrm{~d}$ show that the wave height, wave period and inflow velocity were the dominant factors on the varience of the loads. Since these parameters are influential in regards to the load fluctuation it is believed that they will be design drivers for the fatigue loading. Also, since they cannot be controlled in the same manner as $\Omega_{\mathrm{r}}$ and $\theta$, site selection and characterisation will be of great importance to ensure device longevity.

The results for Case 2 of the sensitivity analysis in Figure 5 showed that the rotor radius $R$ and blade pitch $\theta$ were the primary parameters on both the mean loads and load variance in the geometry domain. A possible explanation for the rotor radius influence on the variance is that the longer blades in a larger turbine sweep a larger section of the water column, thus exposing them to a greater range of inflow velocities.

The results for Case 3 in Figure 6 show the same parameters having the largest influence on the eccentric loading as in the previous cases, with the exception of the blade's initial azimuth position, $\phi_{0}$, having a greater sensitivity score. The arc angle of the swept eccentricity vector in Figure 6a shows greatest sensitivity to the blade initial azimuth, $\phi_{0}$, the rotor angular velocity, $\Omega_{\mathrm{r}}$, and the wave period, $T$ - parameters which are all related to the relative phasing between rotor and incident waves. This result indicates that the synchronisation between the rotating blades and the passing waves may have a large influence on the eccentric load variance and load directionality. The blade pitch also shows high importance on the arc angle which was not expected, and a further analysis of this is shown in Section 4.

When considering the maximum magnitude of the thrust eccentricity in Figure $6 \mathrm{~b}$, the rotor radius, blade pitch and wave height were identified as the primary parameters. Again, the sensitivity towards the turbine radius is explained by the greater velocity gradient across the turbine plane, which gives rise to a larger eccentricity vector, and a similar reason is given for the influence of the wave height. The pitch angle's large influence on the eccentricity magnitude is hypothesised to be caused by the parameter changing how close to or far away from the optimum angle of attack the blades operate; having the blades operating in a range close to the stall region for example will induce a greater and more unpredictable load range, and thus higher eccentricity.

\section{Analysis of Primary Parameters}

Based on the results from the sensitivity analysis, the 8 most influential parameters out of the 13 investigated were selected for a detailed analysis of their influence on the rotor out-of-plane bending loads. The parameters selected for further analysis were the current vorticity distribution, the rotor angular velocity, the blade root pitch, the rotor radius, the blade initial azimuth position, the surface velocity, the wave period and the wave height. 
The purpose of the analysis was to show the effect that the variation of a single input parameter had on the magnitude and directionality of the eccentric rotor loadpattern caused by the non-uniform blade-loading; this would ultimately inform on which parameters had the largest impact on the internal loading of a TST.

The analysis was performed by first defining a base-case turbine load environment where each parameter assumed the mean values of the ranges presented for Case 3 of the sensitivity analysis (shown in Table 1 ). The base-case thus consisted of a $12.5 \mathrm{~m}$ radius turbine operating in a constant gradient velocity profile with a $3.25 \mathrm{~m} / \mathrm{s}$ surface velocity etc.

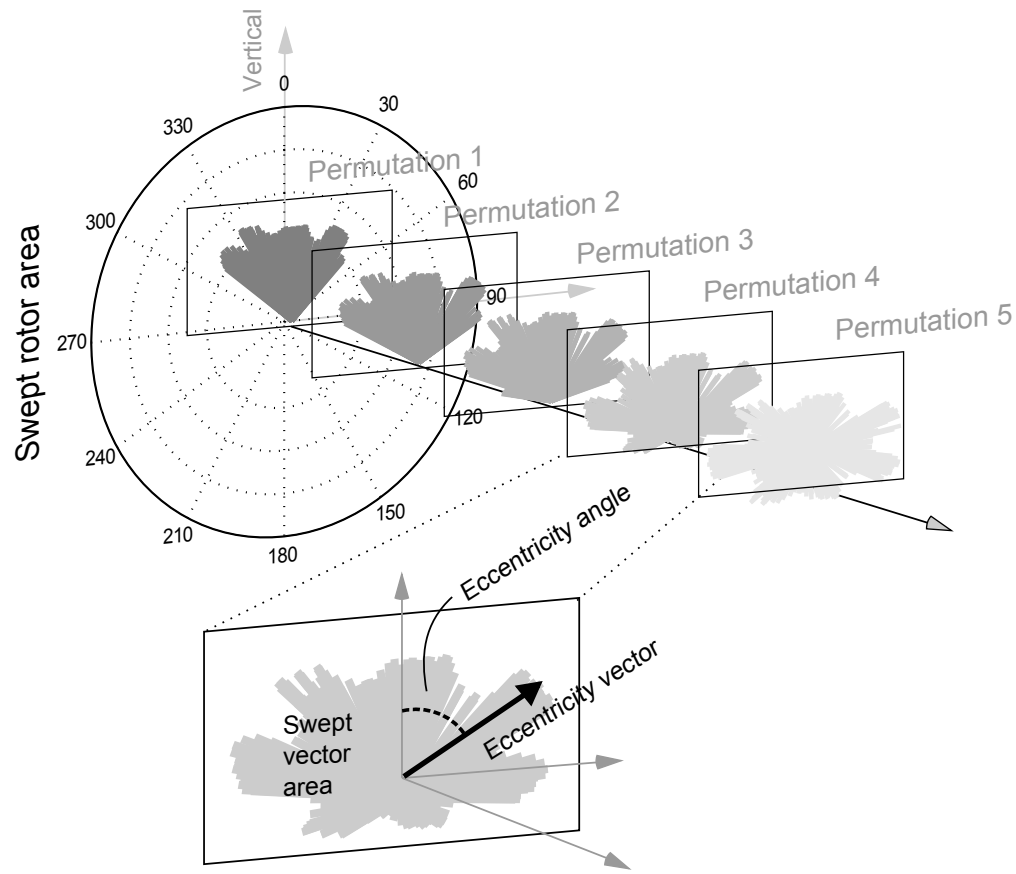

Figure 7: Illustration of the method of presentation of the results of the detailed analysis of the primary parameters. The figure shows the superposition of the five permutations of one single variable, while the others are kept constant. This presentation allows the effect of the parameter variation on the eccentricity pattern to be seen in a comparative and qualitative way.

Each of the investigated parameters were then varied over the ranges defined in Table 1 in five uniform increments, keeping all other parameters constant. The results of each permutation were then presented as the polar plot of the eccentricity vectors graphed over two wave periods and having the five permutations superimposed for visual comparison as illustrated in Figure 7. This allowed both the variations in eccentricity magnitude and angular distribution to be compared for each parameter change. Shown below the polar plots were the corresponding out-of-plane bending moment signals for one blade. Each curve in the sub-figures represents the loads for one parameter variation, giving an indication of how much the total loads on the whole turbine varied with each permutation. 

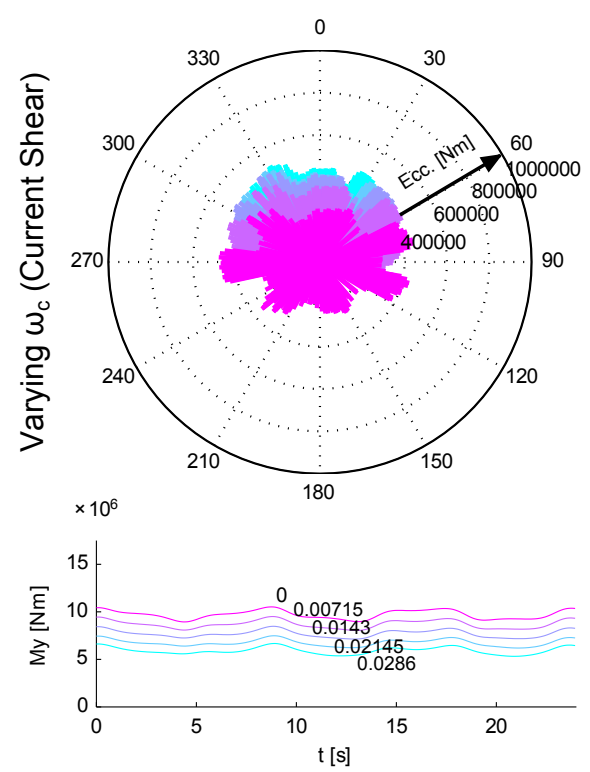

(a)
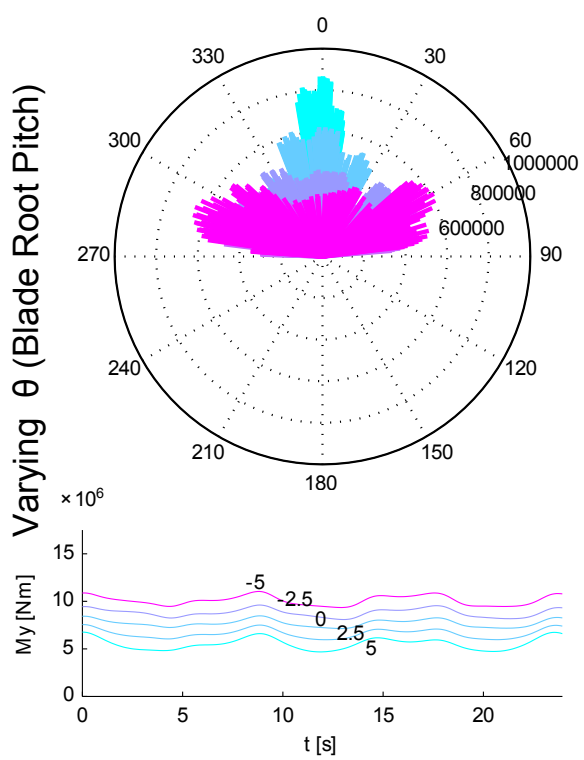

(c)

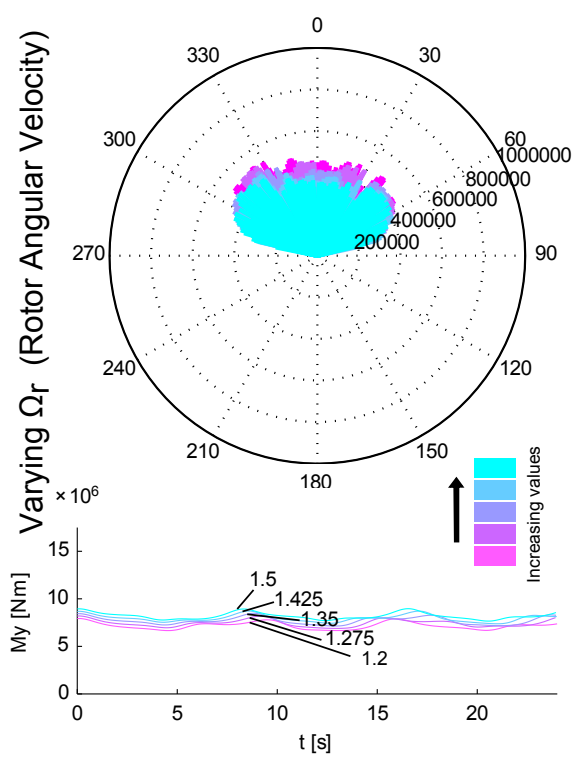

(b)
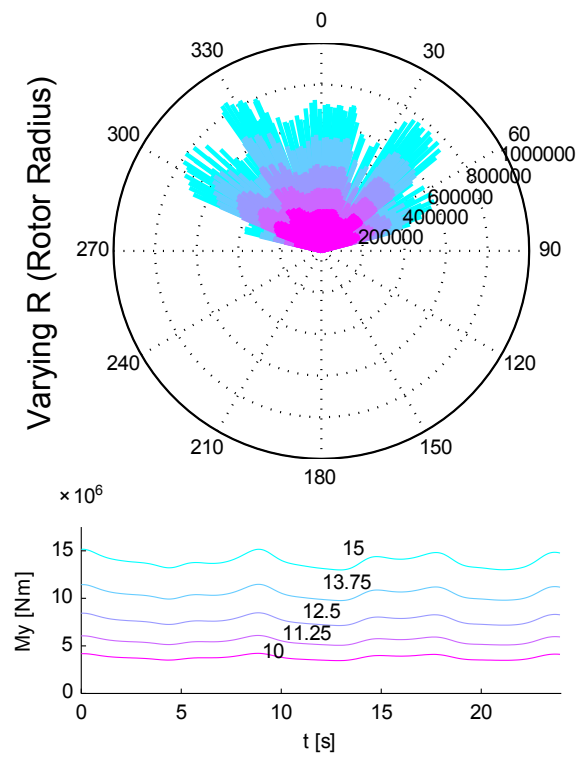

(d)

Figure 8: Results from primary variable analysis showing polar plots of the eccentric load patterns and single blade bending moments $\left(M_{y}\right)$ for different single variable permutations. The parameters varied are: varying shear current (a), varying rotor angular velocity (b), varying blade root pitch (c), varying rotor radius $(d)$. 


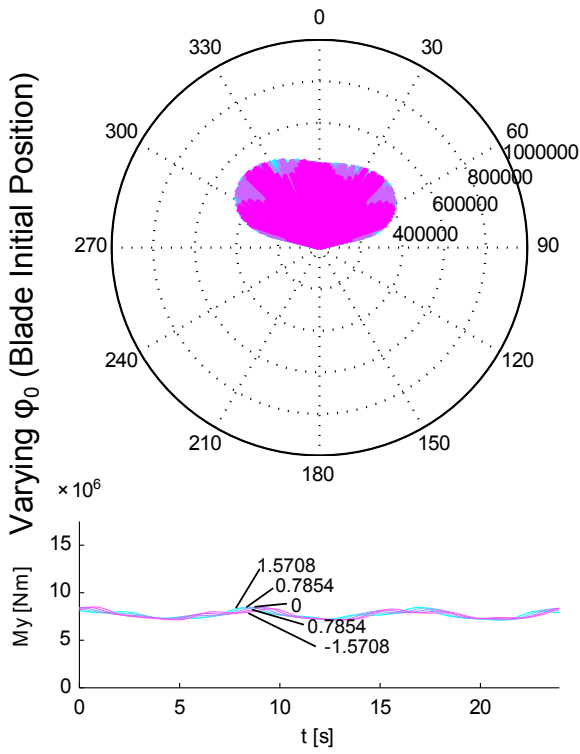

(a)
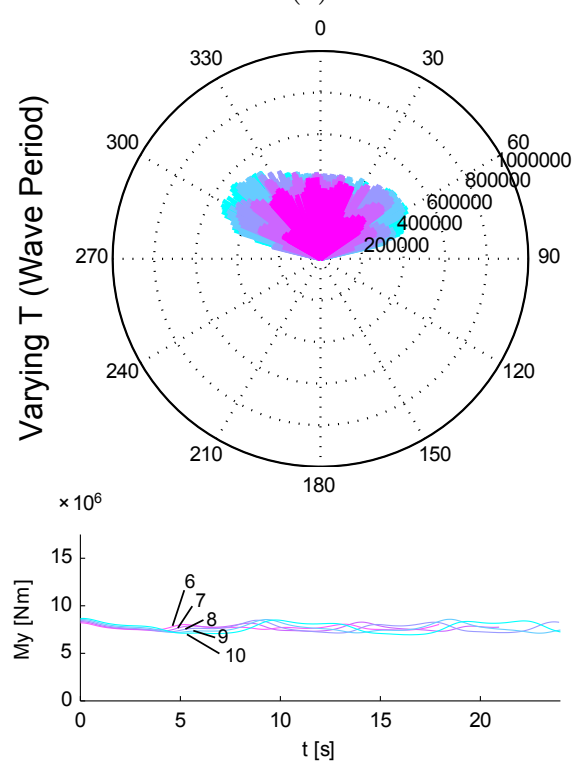

(c)

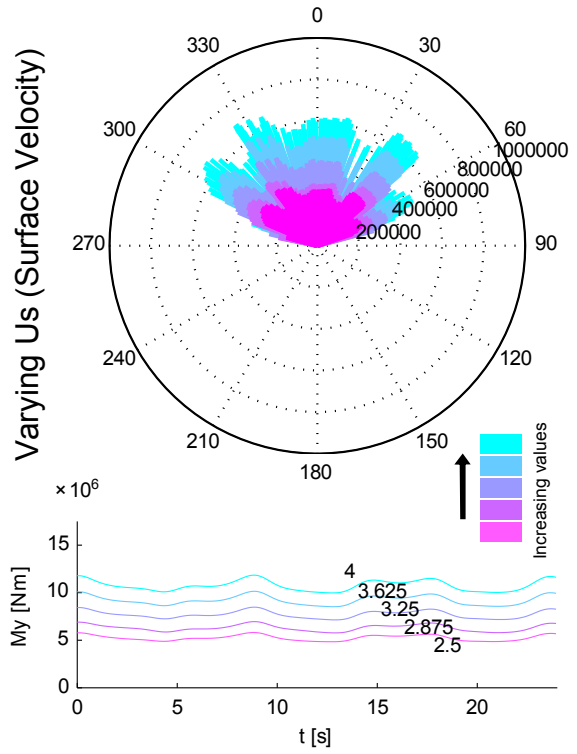

(b)
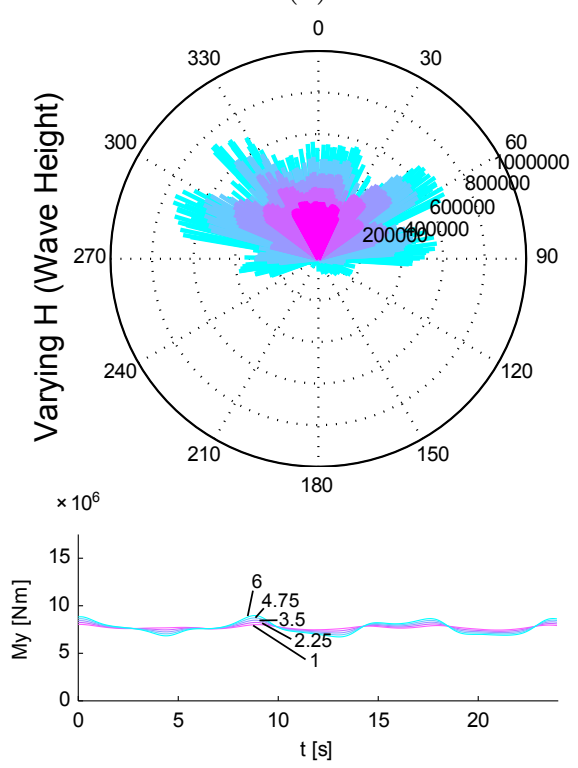

(d)

Figure 9: Results from primary variable analysis showing polar plots of the eccentric load patterns and single blade bending moments $\left(M_{y}\right)$ for different single variable permutations. The parameters varied are: varying blade initial azimuth position (a), varying surface velocity (b), varying wave period (c), varying wave height $(\mathrm{d})$. 


\subsection{Primary Variable Analysis Results and Discussion}

The results of the detailed analysis of the primary parameters' relations to the eccentricity are shown in Figures 8 - 9. It can be seen that the eccentric rotor bending moment loads transmitted to the shaft were significant and could reach values as high as approximately $8 \times 10^{5} \mathrm{Nm}$ as shown in Figure 8 d. This bending load could be equated to a point load of approximately 6.5 tonnes applied at the turbine blade tip of $12.5 \mathrm{~m}$ radius.

Figure 8a shows the variation of the eccentricity vector induced by the variation of current shear steepness. It can be seen that as the current shear steepness increases, the eccentricity pattern increases in magnitude towards the 'upward' direction due to the greater velocity distribution towards the top of the rotor. However, as can be seen in the lower graph of Figure 8a, the loading on the individual blades decreased for increasing current shear as the current shear was generated by decreasing the bottom inflow velocity. This implied that a site having strong current shear steepness would generate lower loads averaged over the entire rotor area while increasing the eccentricity magnitude due to the velocity gradient.

Figure $8 \mathrm{a}$ is also the only case where a 'downward' directionality of the eccentric loads can be seen in the case where the current shear is zero, i.e uniform inflow. This phenomenon will occur whenever the trough of a large enough wave passes a turbine operating in a uniform current since the top of the rotor is then exposed to the largest velocity reversal from the wave, creating a inverted load gradient over the rotor-plane.

As mentioned in Section 3.3, the blade root pitch setting showed a large influence on the eccentricity magnitude which can be seen in Figure 8c. Here, the eccentricity magnitude increases and the arc angle of the swept area decreases with increasing values of blade pitch. Similarly to the current shear results, the eccentricity magnitude was also increased while the loads on the individual blades decreased, as can be seen in the bending moment plot of Figure 8c. This latter finding is interesting from a condition monitoring aspect of a turbine since, if the load signal from a single blade is used to monitor the turbine's loads, one might unintentionally generate higher loads on the drivetrain by reducing the time-averaged load on the blade.

The results from the rotor radius variations in Figure 8d showed a predictable loadchange where the eccentricity pattern was 'scaled' geometrically with increasing radius. This parameter variation also showed the largest change in the individual blade loads and also produced a significant increase in the load signal variance as predicted by the sensitivity analysis. The results from the surface velocity variations show a similar geometrical change in the load pattern although it was less severe in magnitude, as shown in Figure 9b.

As mentioned in the previous section, the wave period was one of the parameters that had a strong impact on the arc angle of the eccentricity. This is visualised in Figure 9c where the larger periods show a larger angular range and a larger eccentricity magnitude towards the extreme angles. The case where the wave period was 10 s was where the rotor, having a rotational period close to $5 \mathrm{~s}$, may have synchronised with the incoming wave train giving rise to the largest eccentricity magnitudes. 


\section{Conclusions}

Based on the results from both the sensitivity analysis and the detailed analysis of the primary parameters, it is believed that the current shear, blade root pitch, rotor radius, surface velocity and wave height will be the most influential parameters in terms of the loading on a tidal turbine. It is clear that these parameters have a large impact on the magnitudes of the loads and their angular distributions, where the latter was found to be larger than reported by Tatum et al. [3] in most cases investigated as shown in Figures 8 - 9. During the structural design stage of a TST, it would be beneficial to investigate the effect that the expected angular spread of the eccentric loads would have on the fatigue life of the drivetrain components, since it may be more challenging to accommodate for a highly spatially varying load. On the other hand, a load pattern that is more evenly distributed may alleviate the localised fatigue damage that may occur on a component that is constantly subjected to the eccentric bending stresses from one direction.

The sensitivity analysis on the turbine loads revealed that some parameters, such as the wave height, had a large impact on the variance of the loads but did not have a significant impact on the loading mean value. These parameters will be important to consider when investigating the fatigue life of a device.

It is curious to note that the hub height of the turbine seems to have a small impact on the turbine loads which is contradictory to the findings of Milne et al. [12]. One explanation to this may be that the difference in height needed to show a variation in the loads was large, meaning that the sensitivity of the loads to this parameter was not significant. Another explanation may be that the investigated range of the hub height did not span a great distance, since the BEMT model could not incorporate the effects of free-surface interactions for a shallow turbine immersion.

Since the rotor radius and blade pitch were shown to be among the most influential on the turbine loads, it would be possible to engineer for load mitigation if the other inflow conditions are known with high confidence. This study could be extended to include metrics of the power capture for each parameter variation, which would make it possible to find a most economic design trade-off between longevity and power capture.

The results for the analysis of the primary parameters' effect on the eccentricity showed that the eccentric thrust loads transmitted to a TST's shaft from the rotor were appreciable and varied drastically depending on the turbine's inflow conditions, geometrical design and operating modes. It is believed that when transmitted to a turbine's drive train, these non-axial loads will have a detrimental effect on internal components such as bearings and seals, which are usually not designed to withstand large variations in load-magnitude and direction. The results show that there is a clear need to investigate the role of the thrust eccentricity on the turbine's internal structural components when considering ultimate and fatigue load calculations.

In conclusion, this paper has made an attempt to shed light on the complicated interactions between the various input parameters and the load outputs on a TST operating in the unsteady marine environment. Although the rotor radius, current shear, blade root pitch, surface velocity and wave height have been identified as the primary parameters, there is still many unknown interactions between the other parameters which may play an important role in other turbine working-conditions. It is therefore recommended that a sensitivity analysis is performed on all of the known input parameters prior to the deployment of a device, including the effects of ambient flow turbulence, to insure 
that the relationships between the input variables and the output loads of the system are fully known for each specific deployment. Also, it is advisable that when determining load mitigation strategies for TSTs that developers should consider the increase in eccentricity that arises from increasing the load range of the blades as shown in Figure $8 \mathrm{a}$ and ??.

Future work will include fatigue calculations on the internal components of a TST based on the results of the identified primary parameters with the inclusion of the eccentric degree of freedom of the loads.

\section{Acknowledgements}

The authors would like to express their gratitude to EPSRC for funding this project through SuperGen UKCMER.

\section{References}

[1] M. Gough, F. Fortune, F. Johnson, Meygen tidal energy project phase 1 environmental management plan: Construction works, http://www.gov.scot/Resource/0048/00482942.pdf, [Accessed: 201511-09] (2015).

[2] S. D. Weller, P. R. Thies, T. Gordelier, L. Johanning, Reducing reliability uncertainties for marine renewable energy, Journal of Marine Science and Engineering 3 (4) (2015) 1349-1361.

[3] S. Tatum, C. Frost, M. Allmark, D. O'Doherty, A. Mason-Jones, P. Prickett, R. Grosvenor, C. Byrne, T. O'Doherty, Wave-current interaction effects on tidal stream turbine performance and loading characteristics, International Journal of Marine Energy (2015) .

[4] P. Galloway, Performance quantification of tidal turbines subjected to dynamic loading, Ph.D. thesis, University of Southampton (2013).

[5] A. Bahaj, W. Batten, G. McCann, Experimental verifications of numerical predictions for the hydrodynamic performance of horizontal axis marine current turbines, Renewable Energy 32 (15) (2007) $2479-2490$.

[6] N. Barltrop, K. S. Varyani, A. Grant, D. Clelland, X. P. Pham, Investigation into wave- current interactions in marine current turbines, Proceedings of the Institution of Mechanical Engineers, Part A: Journal of Power and Energy 221 (2) (2007) 233-242.

[7] B. Gaurier, P. Davies, A. Deuff, G. Germain, Flume tank characterization of marine current turbine blade behaviour under current and wave loading, Renewable Energy 59 (2013) 1-12.

[8] H. Buckland, Wave implementation in blade element momentum theory for modelling tidal stream turbines, in: ACME 2011 Proceedings of the 19 th UK National Conference of the Association for Computational Mechanics in Engineering, 2011.

[9] I. Masters, J. A. C. Orme, A robust blade element momentum theory model for tidal stream turbines including tip and hub loss corrections, Journal of Marine Engineering \& Technology 10 (1) (2011) $25-36$.

[10] G. McCann, Tidal current turbine fatigue loading sensitivity to waves and turbulence - a parametric study, in: Proceedings from 7th European wave and tidal energy conference, 2007.

[11] P. W. Galloway, L. E. Myers, A. S. Bahaj, Quantifying wave and yaw effects on a scale tidal stream turbine, Renewable Energy 63 (2014) $297-307$.

[12] I. Milne, R. Sharma, R. Flay, S. Bickerton, The role of onset turbulence on tidal turbine blade loads, in: Proc. 17th Australasian Fluid Mechanics Conference, Auckland, NZ, 2010.

[13] E. Bossanyi, Bladed for windows theory manual, Garrad Hassan and partners Ltd. (1997).

[14] T. Nevalainen, C. Johnstone, A. Grant, An unsteady blade element momentum theory for tidal stream turbines with Morris method sensitivity analysis, in: Proceedings of the 11th European Wave and Tidal Energy Conference, 2015.

[15] M. D. Morris, Factorial sampling plans for preliminary computational experiments, Technometrics 33 (2) (1991) 161-174.

[16] D. Markus, R. Wüchner, K.-U. Bletzinger, A numerical investigation of combined wavecurrent loads on tidal stream generators, Ocean Engineering 72 (2013) 416-428. 
[17] T. Wang, J. Li, Effect of nonlinear wave-current interaction on flow fields and hydrodynamic forces, Science in China Series A: Mathematics 40 (6) (1997) 622-632.

[18] N. Kishida, R. J. Sobey, Stokes theory for waves on a linear shear current, Journal of engineering mechanics 114 (8) (1989) 1317-1334.

[19] T. Burton, N. Jenkins, D. Sharpe, E. Bossanyi, Wind energy handbook, John Wiley \& Sons Ltd., 2001.

[20] J. Manwell, J. McGowan, A. Rogers, Wind Energy Explained: Theory, Design and Application, John Wiley \& Sons Ltd., 2010.

[21] S. Oye, Tjaereborg Wind Turbine (Esbjerg): First dynamic inflow measurement, AFM notat VK 184, Technical University of Denmark, Dept. of Fluid Mechanics, 1991

[22] S. Oye, Tjaereborg Wind Turbine: 4. dynamic inflow measurement, AFM notat, Technical University of Denmark, Dept. of Fluid Mechanics, 1991.

[23] J. Schepers, H. Snel, Final Results of the EU Joule Projects "dynamic Inflow", ECN-RX, Netherlands Energy Research Foundation ECN, 1995.

[24] H. Snel, J. Schepers, Engineering models for dynamic inflow phenomena, Journal of Wind Engineering and Industrial Aerodynamics 39 (1) (1992) 267-281.

[25] H. Snel, J. Schepers, S. E. C. Nederland, Joint investigation of dynamic inflow effects and implementation of an engineering method, Netherlands Energy Research Foundation ECN, 1995.

[26] G. Gaonkar, D. Peters, Review of dynamic inflow modeling for rotorcraft flight dynamics., VERTICA. 12 (3) (1988) 213-242.

[27] J. Whelan, J. Graham, J. Peiro, Inertia effects on horizontal axis tidal-stream turbines, in: the 8th European Wave and Tidal Energy Conference, 2009.

[28] H. Buckland, Combined current, wave and turbulent flows on tidal energy. volume 1, Ph.D. thesis, Swansea University (2013).

[29] D. Doman, R. Murray, M. Pegg, K. Gracie, C. Johnstone, T. Nevalainen, Dynamic testing of a 1/20th scale tidal turbine, in: Proceedings from the Asian Wave and Tidal Energy Conference: Tokyo,Japan, 2014.

[30] D. King, B. Perera, Morris method of sensitivity analysis applied to assess the importance of input variables on urban water supply yield - a case study, Journal of Hydrology 477 (0) (2013) $17-32$.

[31] K. Dykes, A. Ning, R. King, P. Graf, G. Scott, P. Veers, Sensitivity analysis of wind plant performance to key turbine design parameters: A systems engineering approach, in: 32nd ASME Wind Energy Symposium, National Harbor, Maryland, 2014.

[32] L. Ziegler, S. Voormeeren, S. Schafhirt, M. Muskulus, Sensitivity of wave fatigue loads on offshore wind turbines under varying site conditions, Energy Procedia 80 (2015) 193 - 200, 12th Deep Sea Offshore Wind R\&D Conference.

[33] A. Saltelli, S. Tarantola, F. Campolongo, M. Ratto, Sensitivity Analysis in Practice: A Guide to Assessing Scientific Models, John Wiley \& Sons Ltd., 2004. 